\title{
Failed Tracheal Intubation in a Child with Unrecognized Hunter's Syndrome Having Emergency Surgery
}

The Editor,

Sir,

We report a child with unanticipated Hunter's syndrome who, while under general anesthesia for an emergency surgery, suffered from an emergency difficult airway. As the child showed limited cooperation, general anesthesia was chosen. After anesthesia induction, attempts at intubation using bronchofiberscope or Macintosh laryngoscope were performed, and finally the attempts to intubate the trachea were failed. Other attempts using different laryngeal mask airway (LMA) and intubating laryngeal mask were equally unsuccessful either, thus resulting in can't intubation and can't ventilate. Because of the intubation failure, an emergency cannula cricothyroidotomy was completed rapidly. Considering there were difficulties in correctly identifying the cricothyroid membrane due to the abnormal neck anatomy, we promptly used ultrasound guidance to locate the puncture point.

Hunter's syndrome is characterized by progressive pathological lysosomal storage of glycosaminoglycans (GAGs) in nearly all cell types, tissues and organs. In patients with Hunter's syndrome, oropharyngeal and tracheo-bronchial deposition of GAGs leads to severe airway obstruction due to macroglossia, supraglottic narrowing, and tracheomalacia. This obstructive anatomy and physiology leads to sleep apnoea and airway obstruction. The high prevalence of airway obstruction poses a high anesthetic risk to these patients. Typical anesthetic problems include airway obstruction after induction or extubation, intubation difficulties or failure, possible emergency tracheotomy, and cardiovascular and cervical spine 
issues. These disorders are uncommon and few anesthetists care for these patients on a regular basis.

In the patients with Hunter's syndrome, intubation is often extremely difficult, although fiberoptic laryngoscopy or bronchoscopy may be useful. Although the LMA has proved to be a useful aid in some difficult cases, the ventilation with the LMA didn't succeed in the child. The reasons might be the obstructive anatomy and physiology in upper airway and the local severe edema resulting from the repeating attempts to intubation.

Preoperative evaluation of the upper airway is of the greatest importance, and an emergency response program must be made beforehand carefully in Hunter's syndrome. Ultrasound guidance can identify the cricothyroid membrane when there is difficulty in location, and may improve success possibility and safety when cannula cricothyroidotomy is used.

Keywords: Anesthesia, cricothyroidotomy, difficult airway, Hunter's syndrome

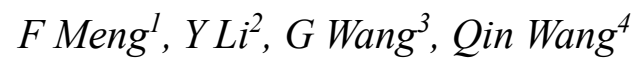

From: 1, 2, ${ }^{4}$ Department of Anesthesiology, Shandong University Affiliated Jinan City Central Hospital, Jinan 250013, PR China. ${ }^{3}$ Department of Anesthesiology, Weifang Medical University, Weifang City 261053, Shangdong Province, PR China. R China, 250013. E-mail: mfj15318816233@163.com

\section{AUTHORS' NOTE}

The authors report no conflicts of interest or funding to disclose. 


\section{REFERENCES}

1. Wraith JE, Scarpa M, Beck M, Bodamer OA, De ML, Guffon N, et al. Mucopolysaccharidosis type II (Hunter syndrome): a clinical review and recommendations for treatment in the era of enzyme replacement therapy. Eur.J.Pediatr. 2008; 167: 267-77.

2. Moores C, Rogers JG, McKenzie IM, Brown TC: Anaesthesia for children with mucopolysaccharidoses. Anaesth.Intensive Care 1996; 24: 459-63.

3. Busoni P, Fognani G. Failure of the laryngeal mask to secure the airway in a patient with Hunter's syndrome (mucopolysaccharidosis type II). Paediatr.Anaesth. 1999; 9: $153-5$.

4. Walker R, Belani KG, Braunlin EA, Bruce IA, Hack H, Harmatz PR, et al. Anaesthesia and airway management in mucopolysaccharidosis. J.Inherit.Metab Dis. 2013; 36: 211-9.

5. Kodani Y, Matsuura T, Mori T, Nishikawa K, Asada A. [Anesthesia for three patients with Hunter syndrome]. Masui 2011; 60: 846-9.

6. Darmon JY, Rauss A, Dreyfuss D, Bleichner G, Elkharrat D, Schlemmer B, et al. Evaluation of risk factors for laryngeal edema after tracheal extubation in adults and its prevention by dexamethasone. A placebo-controlled, double-blind, multicenter study. Anesthesiology 1992; 77: 245-51.

7. Tellez DW, Galvis AG, Storgion SA, Amer HN, Hoseyni M, Deakers TW. Dexamethasone in the prevention of postextubation stridor in children. J.Pediatr. 1991; 118: 289-94. 
8. Jain D, Gandhi K. Comparing sugammadex and neostigmine reversal of neuromuscular blockade in laparoscopic surgery. Anaesthesia 2013; 68: 306-7. 